\title{
Anestesia intra-articular com ropivacaína para diagnóstico de claudicação em cães
}

\author{
[Intra-articular anesthesia with ropivacaine for diagnosis of claudication in dogs]
}

\section{"Artigo Científico/Scientific Article"}

\author{
Kássia Fernanda Araújo Damasceno ${ }^{1 *}$, Kleyton Domingos de Melo $^{2}$, Maria Raquel de Almeida ${ }^{1,2}$, \\ Eduardo Alberto Tudury ${ }^{1,2}$
}

\begin{abstract}
${ }^{1}$ Departamento de Medicina Veterinária, Universidade Federal Rural de Pernambuco, Recife-PE, Brasil.
${ }^{2}$ Programa de Pós-Graduação em Medicina Veterinária, Universidade Federal Rural de Pernambuco (UFRPE), Recife-

PE, Brasil.

*Autor para correspondência/Corresponding author: E-mail: kassiafernadaad@ hotmail.com
\end{abstract}

\begin{abstract}
Resumo
A anestesia intra-articular na medicina veterinária é uma prática recente e usada para alívio da dor em cirurgias artroscópicas, entretanto uso para o diagnóstico de claudicação na clínica médica de cães é infrequente. A técnica pode ser realizada em qualquer articulação como forma de localizar a fonte da claudicação. Nesta pesquisa foram avaliados 20 animais, os quais foram identificados claudicando por dois avaliadores veterinários, visualmente e por meio de filmagem (normal e "slow motion"), antes e após a administração intra-articular de ropivacaína $0,5 \%$ na dose de $0,5 \mathrm{mg} / \mathrm{kg}$. Com $48 \mathrm{~h}$, sete e 30 dias após a administração não foram verificados a ocorrência de agravamento da claudicação assim como efeitos sistêmicos indesejáveis. Os resultados permitem sugerir que a anestesia intra-articular oferece a possibilidade de determinar que o problema é situado numa única articulação, assim como além de haver uma articulação afetada outras articulações ou estruturas não passíveis de anestesia intra-articular podem estar afetadas. Em 10 animais (50\%), o diagnóstico final correspondeu ao da pré-anestesia intra-articular, em cinco confirmou-se a suspeita inicial, mas foi identificada outra afecção concomitante e em três casos houve mudança de diagnóstico. Conclui-se que a ropivacaína quando administrada em cães pela via intra-articular nessa dosagem não tem efeitos deletérios e funciona como método de diagnóstico seguro e eficiente para localizar a fonte da claudicação nesses pacientes.
\end{abstract}

Palavras-chave: Caninos; articulação; dor; ortopedia; anestésico local.

\begin{abstract}
Intra-articular anesthesia in veterinary medicine is a recent practice and is used for pain relief in arthroscopic surgeries, being its use for diagnosis of claudication of infrequent frequency in the medical clinic of dogs. It can be done at any joint as a way of locating the source of claudication. In this study, 20 animals were evaluated, which were examinated by two veterinary evaluators, visually and by means of filming (normal and slow motion), before and after the intra-articular administration of ropivacaine $0.5 \%$ in dose of $0.1 \mathrm{ml} / \mathrm{kg}$ $(0.5 \mathrm{mg} / \mathrm{kg})$, aseptically. In post administration with $48 \mathrm{~h}, 7$ and 30 days the occurrence of worsening of claudication, as well as systemic undesirable effects were not verified. Previous radiographs were performed in search of the radiographic signs present. The results allow to suggest that intra-articular anesthesia offers the possibility to determine that the problem is situated in a single joint, as well as that in addition to having an affected joint other joints or structures not subjected to intra-articular anesthesia may be affected. In 10 animals $(50 \%)$, the final diagnosis corresponded to that of intra-articular pre-anesthetic. Five confirmed whether the initial suspicion was found to be associated with another condition. However, there was a change of diagnosis in three dogs. It is concluded that ropivacaine when administered intra-articularly in this dosage has no deleterious effects and serves as a safe and efficient diagnostic method to locate the source of claudication in dogs.
\end{abstract}

Keywords: Canine; joint; pain; orthopedics; diagnosis; local anesthetic. 


\section{Introdução}

Um dos principais sinais clínicos que são visíveis quanto ao acometimento da articulação em cães é a presença de claudicação, que deve ser diferenciada quanto à sua origem (Piermattei et al., 2009). A compreensão da locomoção do cão através do exame de inspeção é de fundamental importância para diagnosticar condições músculoesqueléticas e neurológicas. Por isso, antes do exame ortopédico ou neurológico, a observação da marcha deve ser realizada, atentando tanto para caminhada como para o trote, devendo ser efetuada em uma superfície plana, inclusive com utilização de escadas, a fim de não passar despercebidas as alterações mais sutis (Carr e Dycus, 2016).

O diagnóstico da claudicação no cão é baseado na história, inspeção, palpação e radiografias sequenciais da região suspeita. Em alguns cães, é difícil determinar a localização do problema devido à ausência de alterações palpáveis, ou devido a uma resposta de dor não confiável. Além disso, a radiografia simples nem sempre pode ser usada para confirmar a localização do problema na articulação suspeita, porque as alterações radiográficas podem ser discretas ou mesmo ausentes (Van Vynckt et al., 2012).

O diagnóstico de claudicação em cães com o uso de anestesia intra-articular teve como base as aplicações para fins diagnósticos e analgésicos póscirúrgicos em grandes animais. O uso de novos fármacos para fins diagnósticos pode levar a uma maior precisão quanto à interpretação da localização e melhora do grau de claudicação em articulações acometidas nos pequenos animais, em especial caninos (Van Vynckt et al., 2010; Van Vynckt et al., 2012). Na medicina veterinária, a anestesia intra-articular também é utilizada para analgesia em cirurgias artroscópicas (Van Vynckt et al., 2012).

As avaliações de alívio da dor pós-cirúrgica em medicina humana levaram ao desenvolvimento de várias técnicas anestésicas, incluindo anestesia multimodal (Danieli et al., 2012). Baseando-se nisso, evidencia-se a necessidade de se aprimorar algumas técnicas anestésicas em animais, além de estas poderem servir de base para novos estudos (Joshi et al., 2000).

A anestesia intra-articular oferece uma forma simples e rápida de identificar a articulação dolorosa. A artrocentese é realizada sob sedação, e após a recuperação confirmatória de líquido sinovial, um anestésico local é injetado. Consecutivamente, o cão é observado para avaliar o efeito do fármaco sobre sua claudicação. A anestesia intra-articular pode resolver temporariamente a claudicação causada por uma variedade de lesões, como sinovite, erosões da cartilagem, fragmentos osteocondrais e lesões intra-articulares de ligamentos (Van Vynckt et al., 2012).

A dor pós-operatória imediata de cirurgia artroscópica do joelho também pode ser bem controlada com o uso intra-articular de anestésicos locais (Berninger et al., 2018). Apesar da dor pós artroscopia ter sido relatada como moderada e de curta duração, vários estudos evidenciaram sua redução após uso intra-articular de anestésicos locais, sem efeitos adversos (Salomadas et al., 2006), inclusive quando comparada a técnicas analgésicas tradicionais, como bloqueio de nervos periféricos e epidural (Berninger et al., 2018). Para além do benefício pós-opertaório imediato, Eker et al. (2017) demonstraram a analgesia da lidocaína intraarticular por até três meses em pacientes com osteoartrite.

Embora a anestesia intra-articular esteja em uso há pouco tempo na clínica médica de pequenos animais, quando comparado à medicina humana, o uso de alguns anestésicos locais fica limitado devido aos seus efeitos tóxicos, que podem estar presentes tanto local como sistemicamente (Grishko et al., 2010; Rigor e Vilaça, 2013). Quanto aos efeitos locais, é relatada indução de apoptose de condrócitos (Lo et al., 2009) provavelmente devido a uma incompatibilidade química entre os anestésicos locais e o líquido sinovial (Bogatch et al., 2010). Os efeitos são dependentes da dose, concentração, do tempo de uso e do tipo de anestésico local utilizado (Lo et al., 2009; Atik, 2012; Kreuz et al., 2018), sendo que a bupivacaína apresenta maiores efeitos deletérios do que a lidocaína em humanos (Bogatch et al., 2010) e em equinos (Park et al., 2011).

A ropivacaína é um anestésico local aminoamida, de longa duração, estruturalmente similar à bupivacaína, que bloqueia os canais de sódio dependentes de diferenças de voltagem dos receptores aferentes de dor. Tem propriedades farmacocinéticas semelhantes à bupivacaína, porém com propriedades farmacodinâmicas diferentes, como os efeitos vasoconstritores e baixa cardio e condrotoxicidade. Produz menor bloqueio motor e maior afinidade pelas fibras $\mathrm{A} \gamma$ e $\mathrm{C}$, tem peso molecular menor que a bupivacaína, por isso pode ser usada em doses intra-articulares maiores com melhor eficiência clínica (Salomadas et al., 
2006), além de apresentar menores efeitos condrotóxicos (Piper et al., 2011).

Esta pesquisa teve como objetivo avaliar se a ropivacaína, quando injetada em uma articulação lesada, funciona como método de diagnóstico seguro e eficiente para localizar a fonte da claudicação em cães.

\section{Material e Métodos}

Foram avaliados 20 cães (Tabela 1), de variados portes, idade, sexo e raça, apresentando claudicação de origem articular em qualquer um dos quatro membros, excluindo-se animais com fraturas, anquiloses, problemas neurológicos, malformações ou afecções musculares visíveis à inspeção. Cada animal foi submetido a exame clínico e os dados foram anotados em fichas individuais. Os animais foram filmados, quanto à forma de caminhada e trote, e o grau de claudicação foi classificado por dois analisadores veterinários, para posterior comparação após aplicação da anestesia na articulação afetada.

Tabela 1 - Características dos cães participantes da pesquisa.

\begin{tabular}{clccc}
\hline Número & Raça & Sexo & Peso $(\mathbf{K g})$ & Idade \\
\hline 1 & Rottweiler & F & 25 & 8 meses \\
2 & Labrador & M & 31 & 12 anos \\
3 & Poodle & M & 5,3 & 6 anos \\
4 & Labrador & F & 40 & 1 ano \\
5 & Labrador & F & 36 & 4 anos \\
6 & SRD & F & 13 & 5 anos \\
7 & Poodle & F & 12,3 & 10 anos \\
8 & Yorkshire terrier & M & 3 & 10 anos \\
9 & Rottweiler & F & 36 & 5 anos \\
10 & SRD & F & 16 & 5 anos \\
11 & Bulldog inglês & F & 20,4 & 8 anos \\
12 & Labrador & M & 32 & 1 ano \\
13 & Sharpei & F & 16 & 10 meses \\
14 & Labrador & M & 24,4 & 18 meses \\
15 & Rottweiler & M & 34 & - \\
16 & SRD & M & 27,2 & 9 meses \\
17 & SRD & F & 20,2 & 4 anos \\
18 & Pinscher & M & 1,6 & 5 anos \\
19 & SRD & M & 34 & 10,9 \\
20 & Poodle & & & \\
\hline
\end{tabular}

SRD: sem raça definida; M: macho; F: fêmea

A anestesia intra-articular foi antecedida de tricotomia da região, com antissepsia prévia, utilizando-se compressas de gaze estéril, álcool a $70 \%$ e gluconato de clorexidina a $2 \%$. A técnica de punção foi realizada segundo Borer (2006), fazendo uso de luvas e panos de campo, assim como seringas e agulhas, todos estéreis. A punção foi realizada com seringas de $3 \mathrm{ml}$, utilizando-se uma agulha hipodérmica $25 \times 7 \mathrm{~mm}$. O líquido sinovial foi aspirado antes da administração do anestésico local para confirmar se a agulha estava presente na articulação. Foi utilizado como anestésico local ropivacaína $0,5 \%$, na dose de $0,5 \mathrm{mg} / \mathrm{kg}$ (dose mínima utilizada como anestésico local) (Klaumann e Otero, 2013), o que equivale a $0,1 \mathrm{ml} / \mathrm{kg}$ (dose mínima recomendada para anestesia intra-articular) (Fish et al., 2008), a fim de evitar condrotoxicidade ou citotoxicidade.

Após a administração do anestésico local, procedeu-se à flexão e extensão da articulação, objetivando-se espalhar o fármaco por todo o seu interior. Subsequente, foram feitas filmagens semelhantes com duração de cerca de dois minutos com intervalos médios de cinco minutos entre elas, até o período de 30 minutos. O início da filmagem foi com um mínimo de 10 minutos após aplicação da ropivacaína, que corresponde ao período de latência do fármaco. As filmagens foram verificadas por dois analisadores utilizando-se o recurso computacional de "Slow Motion", sendo os graus de claudicação verificados e foi feita a análise comparativa antes e depois da aplicação do anestésico local. Os pacientes foram 
acompanhados com $48 \mathrm{~h}$, sete dias e 30 dias após a administração da anestesia intra-articular, com o propósito de serem observadas sequelas e efeitos clínicos adversos articulares após o uso desse fármaco.

Todos os animais em estudo foram submetidos a exames complementares (dentre eles radiografias), para o diagnóstico quanto à afecção e a análise comparativa associando o tipo de afecção com a melhora ou piora da claudicação, nos períodos citados acima.

Visando observar a ocorrência de alterações sistêmicas decorrentes de efeitos da ropivacaína injetada intra-articular, parâmetros como frequências cardíaca e respiratória, pressão arterial sistólica, temperatura corporal, tempo de preenchimento capilar e coloração das mucosas foram verificados.

Foi confeccionada uma ficha individual para cada animal onde foram transcritos todos os dados referentes ao paciente, assim como os resultados obtidos de todas as análises, a qual serviu para posteriormente comparar o realizado e os resultados do coletivo. Os resultados foram expressos em medidas de frequência (dados enumerativos), ou seja, porcentagens.

\section{Resultados e Discussão}

A metodologia de avaliação dos caninos com claudicação se baseou nas recomendações de Jhonson e Tobias (2017), quanto à utilização de inspeção, exame físico - ortopédico e estudos de imagem, entretanto visando maior precisão na localização da articulação afetada, optou-se por adotar o preconizado por Van Vynckt et al. (2010) e Van Vynckt et al. (2012), cuja interpretação da localização e melhora do grau de claudicação em articulações acometidas nos pequenos animais, em especial caninos, pode ser obtido com a anestesia intra-articular.

O valor diagnóstico da anestesia intraarticular com ropivacaína foi avaliado então em 20 animais, de raças variadas, dez machos e dez fêmeas, com pesos variando de 1,6 a $42 \mathrm{~kg}$ e idades de oito meses a 12 anos. As filmagens, com intervalos de 5 minutos entre elas, até o período de 30 minutos, permitiram a identificação do cessar da claudicação no tempo médio de 15 minutos, com permanência de mais 15 minutos, indicando ser esse o intervalo de tempo ideal para averiguação do grau de claudicação após a anestesia intra-articular.

Nenhum dos pacientes foi submetido à sedação, procedimento recomendado por Van
Vynckt et al. (2012) para realização da artrocentese, já que administração de fármacos sistêmicos poderia ter prejudicado as avaliações de claudicação. Esta decisão aqui tomada discorda do mencionado por esses autores, quando afirmam que a claudicação não é afetada pela sedação. Em todos os casos a aspiração do líquido sinovial, previamente a injeção da ropivacaína, confirmou, como assinalado por esses autores, que a ponta da agulha estava intra-articular.

Cinco dos cães avaliados possuíam inicialmente diagnóstico de displasia coxofemoral, seis de ruptura do ligamento cruzado cranial (RLCC), quatro de luxação patelar, um de luxação coxofemoral, dois de artrite radio-cárpica e outras lesões individuais como: instabilidade e lesão do tendão de inserção do músculo extensor digital longo, artrite séptica do ombro, miopatia do iliopsoas e tenopatia bicipital e instabilidade medial do ombro (Tabela 2).

Em 10 animais (50\%), o diagnóstico final correspondeu ao da pré-anestesia intra-articular. Em sete confirmou-se a suspeita inicial, mas foi descoberta outra afecção concomitante (exemplo: displasia coxofemoral e miopatia de iliopsoas ou estenose lombosacral, RLCC e entorse tarsal, osteoartrose de joelho e fratura patelar). Enquanto isso, houve mudança de diagnóstico em três cães: o diagnóstico artrite do ombro foi substituído pelo de osteossarcoma, o de displasia coxofemoral por síndrome do cão dançarino e o de RLCC por um diagnóstico não estabelecido.

Houve melhora da claudicação em nove animais (45\%), confirmando a articulação afetada, mas em quatro cães não houve melhora devido à existência de comorbidades geradoras de claudicação, como a miopatia do iliopsoas e a estenose lombo-sacral na displasia coxofemoral, fratura patelar e um caso sem conclusão dos motivos. Enquanto isso, Van Vynckt et al. (2012) confirmaram a suspeita da articulação afetada com localização do problema em 166 dos 190 casos (87\%). Em 3,2\% dos pacientes dessa pesquisa, o método não eliminou a claudicação, significando que o problema não estava localizado dentro da articulação injetada. Em 9,5\% dos animais da pesquisa, o teste foi falsamente negativo, significando que o problema era localizado dentro da articulação injetada, mas a claudicação não diminuiu significativamente. No presente estudo, o cão de número 20 , apesar de ter melhorado a dor à palpação articular, manteve sua claudicação sugerindo tratar-se de um falso negativo. Esta é 
uma das principais limitações da técnica, pois cães podem se acostumar a claudicar e, portanto, resultados negativos devem sempre ser interpretados com cuidado, e outras técnicas de diagnóstico devem ser associadas.

Tabela 2. Número do animal, membro afetado, resposta à anestesia intra-articular; diagnóstico inicial e conclusão diagnóstica final.

\begin{tabular}{|c|c|c|c|c|}
\hline Animal & Membro & Melhorou? & Diagnostico prévio & Diagnóstico final \\
\hline 1 & PD e E & Sim & Displasia coxofemoral & Idem \\
\hline 2 & $P D$ e $E$ & Não & Displasia coxofemoral & Idem e miopatia do iliopsoas \\
\hline 3 & PD & Sim & $\begin{array}{l}\text { Luxação patelar, } \\
\text { instabilidade do joelho e } \\
\text { lesão do tendão do m. } \\
\text { extensor digital longo }\end{array}$ & $\begin{array}{l}\text { Achados anteriores e osteoartrose } \\
\text { de joelho }\end{array}$ \\
\hline 4 & $P D$ e $E$ & Não & Displasia coxofemoral & $\begin{array}{l}\text { Idem, síndrome da cauda equina } \\
\text { e miopatia do iliopsoas }\end{array}$ \\
\hline 5 & PD & Não & $\begin{array}{l}\text { Osteoartrose de joelho e } \\
\text { coxofemoral }\end{array}$ & Idem e fratura patelar \\
\hline 6 & PE & Sim & Luxação coxofemoral & Idem \\
\hline 7 & PD & Sim & $\begin{array}{l}\text { Luxação patelar grau II e } \\
\text { crepitação }\end{array}$ & $\begin{array}{l}\text { Achados anteriores e osteoartrose } \\
\text { do joelho }\end{array}$ \\
\hline 8 & $\mathrm{PE}$ & Sim & RLCC & Idem \\
\hline 9 & PD & Não & RLCC & Diagnostico não estabelecido \\
\hline 10 & $\mathrm{AD}$ & Sim & Artrite radio-cárpica & Idem \\
\hline 11 & $\mathrm{AE}$ & Não & $\begin{array}{l}\text { Lesão bicipital e } \\
\text { instabilidade medial do } \\
\text { ombro }\end{array}$ & Idem \\
\hline 12 & PD e E & Não & Displasia coxofemoral & Síndrome do cão dançarino \\
\hline 13 & $\mathrm{AD}$ & Sim & Artrite radio-cárpica & Idem \\
\hline 14 & PD e E & Não & Displasia coxofemoral & Idem e miopatia do iliopsoas \\
\hline 15 & $\mathrm{AD}$ & Não & Artrite séptica do ombro & Osteossarcoma \\
\hline 16 & PE & Sim & $\begin{array}{l}\text { RLCC e luxação patelar } \\
\text { medial }\end{array}$ & Idem \\
\hline 17 & $\mathrm{PE}$ & Não & RLCC e luxação patelar & Idem e entorse tíbio-társica \\
\hline 18 & PD & Sim & RLCC parcial & Idem \\
\hline 19 & PD & Não & $\begin{array}{l}\text { RLCC e miopatia do } \\
\text { iliopsoas }\end{array}$ & Idem \\
\hline 20 & PD & Não & Displasia coxofemoral & Idem \\
\hline
\end{tabular}

Os resultados permitem sugerir que a anestesia intra-articular oferece a possibilidade de determinar que o problema é situado em uma única ou em mais de uma articulação, assim como que além de haver uma articulação afetada outras estruturas não passíveis de anestesia intra-articular podem estar afetadas, como ocorreu frequentemente na displasia coxofemoral acompanhada da miopatia do iliopsoas (Jhonson e Tobias, 2017).

Dezenove cães não apresentaram agravamento da claudicação a curto e longo prazo após aplicação intra-articular de uma única dose (em baixa concentração) do anestésico local, concordando com a afirmação de Piper et al. (2011) de que a ropivacaína tem baixa condrotoxicidade. A exceção ocorreu em um animal que exibiu, após 30 dias, artrite purulenta do joelho testado, apesar de terem sido realizadas todas as medidas de assepsia que o procedimento requer (tricotomia, antissepsia, uso de luvas e material estéril). Por tratar-se de um animal submetido à cirurgia de osteotomia de nivelamento do platô tibial (TPLO), em que isto não é infrequente (Jhonson e Tobias, 2017), sete dias após a injeção intra-articula, não é seguro atribuir este infortúnio à esta última. Com remoção dos implantes e uso de antibiótico, o processo séptico foi debelado. Em todos os cães o líquido sinovial puncionado se apresentava turvo e/ou ligeiramente hemorrágico, mas não purulento, achado compatível com o relatado nas artropatias por Piermattei et al. (2009) e Jhonson e Tobias (2017).

Nenhum dos cães exibiu sinais de cardiotoxicidade nem sintomas neurológicos, na hora nem após exame, seguramente pelo uso de uma única aplicação, com baixas dose e 
concentração, como já tinha sido sugerido por Di Salvo et al. (2015).

\section{Conclusão}

A anestesia intra-articular pode ser empregada como forma de identificar a localização de afecções que levam à claudicação em cães, entretanto a técnica não é $100 \%$ eficiente pois outras articulações e transtornos extraarticulares podem estar envolvidos. Quando a claudicação desaparece imediatamente após o procedimento, esta mudança assinala ser a articulação em estudo o único desencadeante.

\section{Conflito de Interesse}

Os autores declaram não haver conflito de interesses.

\section{Comitê de Ética}

Comissão de Ética no Uso de Animais (CEUA) da Universidade Federal Rural de Pernambuco (UFRPE) licença no ${ }^{-124 / 2017 . ~}$

\section{Referências}

Atik, O.S. Is single-dose local anesthetic chondrotoxic? Eklem Hastalik Cerrahisi, 23(2):111-2, 2012.

Berninger, M.T.; Friederichs, J.; Leidinger, W.; Augat, P.; Bühren, V.; Fulghum, C.; Reng, W. Effect of local infiltration analgesia, peripheral nerve blocks, general and spinal anesthesia on early functional recovery and pain control in unicompartmental knee arthroplasty. BMC Musculoskelet Disord, 24;19(1):249, 2018

Bogatch, M.T.; Ferachi, D.G.; Kyle, B.; Popinchalk, S.; Howell, M.H.; Ge, D.;You, Z.; Savoie, F.H. Is chemical incompatibility responsible for chondrocyte death induced by local anesthetics? American Journal of Sports and Medicine, 38(3):520-6, 2010.

Borer, K. Local analgesic techniques in small animals. In Practice 28, 200-207, 2006

Carr, B.J.; Dycus, D.L., Canine gait analysis; today's veterinary practice, 2016. P. 93-100.

Danieli, M.V.; Neto, A.C.; Herrera, P., Bupivacaina ou bupivacaina e morfina intraarticular pós reconstrução do LCA, Acta Ortopédica Brasileira, 20(5), 2012

Di Salvo, R; Bufalari, A; De Monte, V; Cagnardi, P; Marenzoni, M.L.; Catanzaro, A; Vigorito, V; Della Rocca, G. Intra-articular administration of lidocaine in anaesthetized dogs: pharmacokinetic profile and safety on cardiovascular and nervous systems. Journal of Veterinary Pharmacology and Therapeutics, 38(4):350-6, 2015.

Eker, H.E.; Cok, O.Y.; Aribogan, A.; Arslan G. The efficacy of intra-articular lidocaine administration in chronic knee pain due to osteoarthritis: A randomized, double-blind, controlled study; Anesthesia Critical Care and Pain Medicine, 23p., 2017

Fish, R.E.; Brown, M.J; Danneman, P.J.; Karas, A.Z. Anesthesia and analgesia in laboratory animals; American college of laboratory animal medicine series; Elsevier, $2^{\mathrm{a}} \mathrm{ed}, \mathrm{p}$. 379.; 2008

Grishko, V.; Xu, M.; Wilson, G.; Albert, W. Apoptosis and mitochondrial dysfunction in human chondrocytes following exposure to lidocaine, bupivacaine, and ropivacaine. The Journal of Bone and Joint Surgery, 92(3): p. 609-18. 2010.

Jhonson, S.A; Tobias, K M. Veterinary surgery small animal. 2 ed. Elsevier, 2017, 6755p.

Joshi, W.; Reuben, S.S.; Kilaru, P.R.; Sklar, J.; Maciolek, H. Postoperative analgesia for outpatient arthroscopic knee surgery with intraarticular clonidine and/or morphine. Anesthesia and Analgesia, 90:1102-1106, 2000.

Klaumann, P.R.; Otero, P.E. Anestesia locorregional em pequenos animais $1 \mathrm{ed}$ São Paulo: Rocca, 2013. p. 01-22.

Kreuz, P.C.; Steinwachs, M.; Angele, P. Singledose local anesthetics exhibit a type-, dose-, and time-dependent chondrotoxic effect on chondrocytes and cartilage: a systematic review of the current literature. Knee Surgery, Sports Traumatology, Arthroscopy, 26(3):819-830, 2018.

Lo, I.K.; Sciore, P.; Chung, M.; Ligang, S.; Boorman, R.B.; Thorton, G.M.; Rattner, J.B.; Muldrew, K. Local anesthetics induce chondrocyte death in bovine articular cartilage disks in a dose- and duration-dependent manner. Arthroscopy, 25(7): 707-715, 2009

Park, J.; Sutradhar, B.C.; Hong, G.; Choi, S.H.; Kim, G. Comparison of the cytotoxic effects of bupivacaine, lidocaine, and mepivacaine in equine articular chondrocytes. Veterinary Anesthesia and Analgesia, 38(2):127-33, 2011

Piper, S.L.; Kramer, J.D.; Kim, H.T.; Feeley, B.T. Effects of local anesthetics on articular 
cartilage. American Journal of Sports and

Medicine, 39(10):2245-53, 2011

Piermattei, D.L.; Flo, G.L.; Decamp, C.E. Ortopedia e tratamento de fraturas de pequenos animais. São Paulo, $4^{\mathrm{a}}$ ed., V.10, p.297-335, 2009.

Rigor, J.S.A.; Vilaça, A. Efeito condrotóxico da injeção intra-articular de fármacos; Instituto de Ciencias Biomedicas Abel Salazar, Universidade de Porto; p. 37; 2013.

Salomadas, E.P. et al. The intra-articular use of ropivacaine for the control of post knee arthroscopy pain. Journal of Orthopaedic Surgery, 1: 17, 2006.
Van Vynckt D.; Polis I.; Verschooten F.; Van Ryssen B. A review of the human and veterinary literature on local anaesthetics and their intra-articular use. Relevant information for lameness diagnosis in the dog. Veterinary and Comparative Orthopaedics and Traumatology, 23: 225-230, 2010.

Van Vynckt, D.; Samoy, Y.; Mosselmans L.; Verhoeven, G.; Verschooten, F.; Ryssen, B.V. The use of intra-articular anesthesia as a diagnostic tool in canine lameness; Vlaams Diergeneeskundig Tijdschrift, 81, 2012. 